\title{
Population Diversity of Leptosphaeria maculans in Australia
}

\author{
Dhwani A. Patel ${ }^{1}$, Manuel Zander ${ }^{1}$, Angela P. Van de Wouw ${ }^{2}$, Annaliese S. Mason ${ }^{3}$, David Edwards ${ }^{4}$ \\ $\&$ Jacqueline Batley ${ }^{4}$ \\ ${ }^{1}$ School of Agriculture and Food Sciences and Centre for Integrative Legume Research, University of Queensland, \\ Brisbane, Australia \\ ${ }^{2}$ School of BioSciences, University of Melbourne, Parkville, Australia \\ ${ }^{3}$ Department of Plant Breeding, Land Use and Nutrition, Justus Liebig University, Giessen, Germany \\ ${ }^{4}$ School of Plant Biology, University of Western Australia, Perth, Australia \\ Correspondance: Jacqueline Batley, School of Plant Biology, University of Western Australia, Crawley, WA 6009, \\ Australia. Tel: 61-8-6488-5929. Email: jacqueline.batley@uwa.edu.au
}

Received: April 21, 2015 Accepted: April 27, 2015 Online Published: May 3, 2015

doi:10.5539/ijb.v7n3p18 URL: http://dx.doi.org/10.5539/ijb.v7n3p18

\begin{abstract}
The fungal pathogen Leptosphaeria maculans, causal agent of blackleg disease, is a primary cause of canola (Brassica napus) crop loss in Australia. Expanding our knowledge of the occurrence of this pathogen in Australia will provide valuable insights into developing methods of resistance against it. In this study, we examine the population diversity of L. maculans in Australia using single nucleotide polymorphisms (SNPs). An Illumina GoldenGate 384 SNP assay was developed and used to genotype 59 blackleg isolates collected from across Australia, in different years and from different stubble sources. Limited linkage disequilibrium, absence of significant clustering in the principal component analysis and a mixed dendrogram suggest that the Australian $L$. maculans population as a whole is panmictic. Some evidence of clonality concentrated in each state was also observed. There was a lack of correlation between SNP haplotypes, stubble cultivar and year of collection. These results suggest a high rate of sexual reproduction and evolutionary diversification in the pathogen. These features could enable the pathogen to overcome resistance and continue to cause disease in Brassica crops. Analysis of these fungal population isolates will help shed some light on evolution and pathogenicity questions in this important crop pathogen.
\end{abstract}

Keywords: Leptosphaeria maculans, blackleg, Single Nucleotide Polymorphisms, goldengate, genetic diversity

\section{Introduction}

The ubiquitous fungal pathogen L. maculans is the causal agent of phoma stem canker (blackleg) in Brassica napus, B. juncea, B. rapa and B. oleracea: canola, vegetable and mustard crops (West, Kharbanda, Barbetti, \& Fitt, 2001). This ascomycete was first described in 1791 by Tode and 1849 by Desmaziéres (Gout, Eckert, Rouxel, \& Balesdent, 2006). Severe epidemics of blackleg disease occurred in Australia during the 1970s, wiping out the nascent canola industry (Rouxel \& Balesdent, 2005). The 1972 epidemic in Australia caused almost 90\% crop losses, highlighting the susceptibility of canola to L. maculans. Annually, this pathogen causes an average loss of AUD $\$ 100$ million to the Australian economy (Zander et al., 2013). Furthermore, L. maculans isolates present in Australia are classified as highly virulent, able to cause disease even in the more resistant Brassica species: B. juncea, B. nigra and B. carinata (Purwantara, Salisbury, Burton, \& Howlett, 1998).

Daverdin et al. (2012) describe that rapid evolution in pathogens gives rise to new strains to combat crop defences. Maintenance of crop resistance directly depends on the field population size of the pathogen, its evolutionary potential and cropping practices that directly affect its reproductive system (Daverdin et al., 2012). L. maculans can survive as a saprobe in the stubble of infected plants for many years and this is usually favoured by dry hot summers and cold winters (West, Kharbanda, Barbetti, \& Fitt, 2001). During this period, it produces sexual inoculum (ascospores), which can travel from several hundred metres to several hundred kilometres (Travadon et al., 2011) and infect plants followed by asexual spore (conidia) production at the site of infection (Rouxel \& Balesdent, 2005). 
Recent genome sequencing revealed that the L. maculans genome has an isochore-like structure (Rouxel et al., 2011), where the genome is divided into AT and GC-rich blocks, probably caused by the amplification of transposable elements and repeat-induced point (RIP) mutations. The RIP mechanism causes nucleotide substitutions from $\mathrm{C}$ to $\mathrm{T}$ and $\mathrm{G}$ to $\mathrm{A}$ and is a premeiotic repeat-inactivation mechanism specific to fungi that creates genetic diversification in the fungal genome (Rouxel et al., 2011). Fudal et al. (2009) reported that RIP affects the AvrLm6 locus, causing gene inactivation and leading to virulence. Furthermore, isolates have been found to undergo continuous deletions and mutations apart from RIP that lead to further genome diversity. Current approaches to establish blackleg resistance in canola have not been successful in fully controlling this pathogen (Hayward, McLanders, Campbell, Edwards, \& Batley, 2012). Therefore, understanding how this fungus has evolved, diversified and spread in Australia is important in providing information for the breeding and sowing of improved resistant varieties of Brassica.

Population diversity in L. maculans has been examined using a variety of markers. Genetic differences between eastern and western Australian isolates have previously been found using microsatellites and minisatellites, which were attributed to the presence of arid desert between the coasts (Hayden, Cozijnsen, \& Howlett, 2007). Minisatellite markers used to analyse four field populations in France found high levels of gene and genotypic diversity within populations and high gene flow between populations, consistent with randomly mating populations (Gout et al., 2006) Dilmaghani et al. (2012) also used minisatellite markers to show that the $L$. maculans population in Western Canada comprises two genetically distinct populations. A further study implementing fourteen minisatellite markers also found clonal sub populations of this pathogen on B. oleracea in Mexico (Dilmaghani et al., 2013). However, Travadon et al. (2011) found the French L. maculans population to be panmictic. This study also employed minisatellite and microsatellite markers. Other investigations into blackleg population structure have also been conducted using amplified fragment length polymorphisms (AFLPs) (Purwantara, Barrins, Cozijnsen, Ades, \& Howlett, 2000) and restricted fragment length polymorphisms (RFLPs) (Barrins, Ades, Salisbury, \& Howlett, 2004). Single Nucleotide Polymorphisms (SNPs) have recently become a popular choice of molecular marker for population diversity studies, and offer significant benefits in terms of abundance in genomes and ease of high-throughput assessment. SNPs are single base-pair differences between two individuals at a particular locus (Appleby, Edwards, \& Batley, 2009). SNPs can be classified as transitions (C to $\mathrm{T}, \mathrm{G}$ to $\mathrm{A}$ ), transversions ( $\mathrm{C}$ to $\mathrm{G}, \mathrm{A}$ to $\mathrm{T}, \mathrm{T}$ to $\mathrm{G}$ or $\mathrm{C}$ to $\mathrm{A}$ ) and insertions/deletions (indels) of a single base pair. Such molecular markers are good tools to analyse the various processes encompassing the population genetics and evolutionary processes of an organism. These include mating systems, patterns of speciation, dispersal, mutation, migration and selection etc. (Giraud, Enjalbert, Fournier, Delmotte, \& Dutech, 2008; Gout et al., 2006).

The Illumina GoldenGate genotyping assay can be used to simultaneously analyse 384-3072 SNP loci across multiple individuals (Tindall et al., 2010). Previous studies using the Illumina GoldenGate assay have shown that it can be used to reliably score SNPs for genetic analysis (Durstewitz et al., 2010). Furthermore, it is cost-effective and flexible for analysing large numbers of SNPs (Appleby et al., 2009). We applied 384 previously developed $L$. maculans SNPs (Zander et al., 2013) in a GoldenGate assay to analyse 59 Australian L. maculans population isolates collected from different years, regions and cultivars, assessing the diversity of this pathogen across Australia.

\section{Materials and Methods}

\subsection{Fungal Samples}

A total of 59 fungal isolates were analysed using the Illumina GoldenGate assay, this comprised of 96 samples including replicates and controls (Table 1). Isolates were carefully selected to cover a wide range of parameters including region of collection, cultivar grown at collection site, year isolated and $A v r$ gene complement (not shown) (Table 1, Appendix A). The isolates received were either stored in liquid form (agar piece in water) or filter form (filter discs in silica beads). The isolates were grown and genomic DNA extracted as in Zander et al. (2013). The extracted DNA was quantified using a Qubit Fluorometer (Life Technologies, 2013). The reference isolate v23.1.3, (for which the genome sequence is available) (Table 1) (Rouxel et al., 2011) was used for data analysis. 
Table 1. List of L. maculans population isolates used in this study

\begin{tabular}{|c|c|c|c|c|c|c|c|c|}
\hline $\begin{array}{l}\text { As referred } \\
\text { to in text }\end{array}$ & Isolate & Year Cultured & $\begin{array}{l}\text { Species isolated } \\
\text { from }\end{array}$ & Stubble cultivar & Stubble collection site & Country/State & Replicates & Reference \\
\hline Ref & Reference (v23.1.3) & Mid-1990 & & - & - & Europe & N/A & (Rouxel et al., 2011) \\
\hline Lm-1 & 04MGPS021 (21) & 2004 & B. napus & AG-Emblem & Eyre Peninsula & SA & 2 & \\
\hline $\mathrm{Lm}-2$ & 06MGPP041 (41) & 2006 & B. napus & Skipton & Lake Bolac & Vic & 2 & \\
\hline Lm-3 & 04MGPP003 & 2004 & B. napus & TI1 Pinnacle & Geelong & VIC & N/A & \\
\hline Lm-4 & 04MGPP008 & 2004 & B. napus & Unknown & Wonwondah & VIC & N/A & \\
\hline $\mathrm{Lm}-5$ & 04MGPP016 & 2004 & B. napus & AG-Emblem & Bordertown & SA & N/A & \\
\hline $\mathrm{Lm}-6$ & 04MGPP022 & 2004 & B. napus & Grace & Moyhall & SA & N/A & \\
\hline $\mathrm{Lm}-7$ & 04MGPP026 & 2004 & B. napus & Grace & Moyhall & SA & N/A & \\
\hline Lm-8 & 04MGPP035 & 2004 & B. napus & TI1 Pinnacle & Geelong & VIC & N/A & \\
\hline Lm-9 & 04MGPP041 & 2004 & B. napus & Grace & Wonwondah - Pymers & VIC & N/A & \\
\hline Lm-10 & 04MGPP043 & 2004 & B. napus & Grace & Wonwondah - Pymers & VIC & N/A & \\
\hline Lm-11 & 04MGPP045 & 2004 & B. napus & Grace & Wonwondah - Pymers & VIC & N/A & \\
\hline $\mathrm{Lm}-12$ & 04MGPP046 & 2004 & B. napus & TI1 Pinnacle & Laharum & VIC & N/A & \\
\hline Lm-13 & 04MGPP049 & 2004 & B. napus & TI1 Pinnacle & Laharum & VIC & N/A & \\
\hline Lm-14 & 04MGPS006 & 2004 & B. napus & Surpass 400 & Eyre Peninsula & SA & $\mathrm{N} / \mathrm{A}$ & \\
\hline Lm-15 & 04MGPS016 & 2004 & B. napus & Surpass $603 \mathrm{CL}$ & Bordertown -Ballinger & SA & $\mathrm{N} / \mathrm{A}$ & \\
\hline Lm-16 & 04MGPS024 & 2004 & B. napus & ATR-Beacon & Bordertown - Ivan & SA & $\mathrm{N} / \mathrm{A}$ & \\
\hline Lm-17 & 05MGPP002 & 2005 & B. napus & ATR-Beacon & Woseley & SA & N/A & \\
\hline $\mathrm{Lm}-18$ & 05MGPP033 & 2005 & B. napus & Skipton & Yeelana & SA & N/A & \\
\hline Lm-19 & 06MGPP019 & 2006 & B. napus & ATR-Beacon & Wagga Wagga & NSW & $\mathrm{N} / \mathrm{A}$ & \\
\hline $\mathrm{Lm}-20$ & 06MGPP025 & 2006 & B. napus & ATR-Beacon & Wagga Wagga & NSW & $\mathrm{N} / \mathrm{A}$ & \\
\hline $\mathrm{Lm}-21$ & 06MGPS032 & 2006 & B. napus & Surpass 501TT & Keith & SA & $\mathrm{N} / \mathrm{A}$ & \\
\hline $\mathrm{Lm}-22$ & 07VTJH002 & 2007 & B. juncea & $\mathrm{JC} 05002$ & Horsham & Vic & $\mathrm{N} / \mathrm{A}$ & \\
\hline $\mathrm{Lm}-23$ & 07VTJH020 & 2007 & B. juncea & JC05007 & Horsham & Vic & N/A & \\
\hline $\mathrm{Lm}-24$ & D13 & 2009 & B. napus & Hyola50 & Cummins & SA & $\mathrm{N} / \mathrm{A}$ & (Marcroft et al., 2012) \\
\hline $\mathrm{Lm}-25$ & 09SMJ087 & 2009 & B. juncea & EXCEED OasisCL & Kaniva & VIC & $\mathrm{N} / \mathrm{A}$ & \\
\hline Lm-26 & 10SMJ041 & 2010 & B. juncea & EXCEED OasisCL & Tamworth & NSW & $\mathrm{N} / \mathrm{A}$ & \\
\hline $\mathrm{Lm}-27$ & LM300 & 2002 & B. napus & TI1 Pinnacle & Mt Barker & WA & 1 & \\
\hline $\mathrm{Lm}-28$ & LM580 & 2003 & B. napus & ATR-Beacon & Wonwondah & Vic & N/A & \\
\hline $\mathrm{Lm}-29$ & LM592 & 2003 & B. napus & TI1 Pinnacle & Mt Barker & WA & $\mathrm{N} / \mathrm{A}$ & \\
\hline Lm-30 & LM659 & 2003 & B. napus & Hyden & Wongan Hills & WA & $\mathrm{N} / \mathrm{A}$ & \\
\hline $\mathrm{Lm}-31$ & LM661 & 2003 & B. napus & Hyden & Wongan Hills & WA & $\mathrm{N} / \mathrm{A}$ & \\
\hline Lm-32 & IBCN13 & 1991 & B. napus & Unknown & Mt Barker & WA & 1 & (Balesdent et al., 2005) \\
\hline Lm-33 & IBCN15 & 1988 & B. napus & Unknown & Streatham & Vic & 2 & (Purwantara et al., 2000) \\
\hline Lm-34 & IBCN16 & 1988 & B. napus & Unknown & Mt Barker & WA & 2 & (Purwantara et al., 2000) \\
\hline Lm-35 & IBCN17 & 1988 & B. napus & Unknown & Millicent & SA & 1 & (Balesdent et al., 2005) \\
\hline Lm-36 & IBCN18 & 1988 & B. napus & Unknown & Penshurst & Vic & 2 & (Purwantara et al., 2000) \\
\hline Lm-37 & IBCN75 & 1987 & B. napus & Unknown & Mt Barker & WA & 2 & (Purwantara et al., 2000) \\
\hline Lm-38 & IBCN76 & 1987 & B. napus & Unknown & Mt Barker & WA & 2 & (Purwantara et al., 2000) \\
\hline
\end{tabular}




\begin{tabular}{|c|c|c|c|c|c|c|c|c|}
\hline Lm-39 & D8 (M) & 2005 & B. napus & Surpass 501TT & Mt Barker & WA & 2 & (Marcroft et al., 2012) \\
\hline Lm-40 & D9 (M) & 2005 & B. napus & ATR-Beacon & Mt Barker & WA & 2 & (Marcroft et al., 2012) \\
\hline Lm-41 & PHW1223 & 1987 & B. napus & Unknown & Mt Barker & WA & 2 & (Purwantara et al., 2000) \\
\hline Lm-42 & V4 & 1988 & B. napus & Unknown & Numurkah & Vic & N/A & (Van de Wouw et al., 2010) \\
\hline Lm-43 & 35 & 1988 & B. napus & Unknown & Penshurst & Vic & N/A & (Van de Wouw et al., 2010) \\
\hline Lm-44 & 80 & 1988 & B. napus & Unknown & Millicent & SA & N/A & (Van de Wouw et al., 2010) \\
\hline Lm-45 & 89 & 1988 & B. napus & Unknown & Millicent & SA & N/A & (Van de Wouw et al., 2010) \\
\hline Lm-46 & 535 & 2003 & B. napus & TI1 Pinnacle & Lake Bolac & Vic & 1 & \\
\hline Lm-47 & 1245 & 1988 & B. napus & Unknown & Galong & NSW & N/A & (Van de Wouw et al., 2010) \\
\hline Lm-48 & 04S012 & 2004 & B. napus & Surpass603CL & Bordertown & SA & 1 & \\
\hline Lm-49 & $04 \mathrm{~S} 005$ & 2004 & B. napus & Surpass 400 & Eyre Peninsula & SA & 1 & \\
\hline Lm-50 & 04P042 & 2004 & B. napus & Grace & Wonwondah & Vic & N/A & \\
\hline Lm-51 & 05P032 & 2005 & B. napus & Skipton & Yeelanna & SA & N/A & \\
\hline Lm-52 & 06Р039 & 2006 & B. napus & Skipton & Lake Bolac & Vic & 1 & (Van de Wouw et al., 2010) \\
\hline Lm-53 & 06S014 & 2006 & B. napus & Surpass 501TT & Bordertown & SA & N/A & \\
\hline Lm-54 & 06S012 & 2006 & B. napus & ATR-Beacon & Bordertown & SA & 1 & \\
\hline Lm-55 & $06 S 039$ & 2006 & B. napus & Hyola60 & Lake Bolac & Vic & N/A & (Van de Wouw et al., 2010) \\
\hline Lm-56 & 06J085 & 2006 & B. juncea & Unknown & Horsham & VIC & 1 & \\
\hline Lm-57 & 06J095 & 2006 & B. juncea & Unknown & Horsham & Vic & N/A & \\
\hline Lm-58 & $06 \mathrm{~J} 112$ & 2006 & B. juncea & Unknown & Horsham & Vic & N/A & \\
\hline Lm-59 & 04MGPP029 & 2004 & B. napus & TI1 Pinnacle & Geelong & VIC & N/A & \\
\hline
\end{tabular}

Note: VIC-Victoria; NSW-New South Wales; WA-Western Australia; SA-South Australia; Isolate v23.1.3 is the result of a series of in vitro crosses between European field isolates (Balesdent et al., 2001); Not all data on these isolates was available, "-“ denotes an unknown variable. IBCN numbers represent the IDs of "International Blackleg Collection Network” isolates (Marcroft et al., 2012).

\subsection{Illumina GoldenGate assay}

A total of 384 SNPs were selected for the Illumina GoldenGate assay. The SNPs were chosen to cover a range of the 76 supercontigs on which SNPs were predicted, from the list of 21,814 SNPs described in Zander et al. (2013). A designability assessment conducted using the Illumina Assay Design Tool (ADT) scored the 384 SNPs at 0.4 or above, which is deemed a good score for the Illumina GoldenGate assay (Durstewitz et al., 2010). Sample preparation for the Illumina GoldenGate assay was performed according to the Illumina GoldenGate Genotyping Assay guide (According to manufacturer's instructions). The software "Genome Studio" (Illumina Inc., 2013) was used to manually cluster the SNPs into one of the two possible genotype clusters (A and B) for this haploid organism. SNPs that clustered confidently were selected for future data analyses and monomorphic and non-clustering SNPs (did not clearly separate into either the 'A' group or the 'B' group) were eliminated from further analyses, resulting in 214 high-quality SNPs. A sub- set of 193 SNPs was used for linkage disequilibrium (LD) analysis (SNPs monomorphic in all isolates except Lm-1 and Lm-2, the isolates which were used to identify polymorphic SNPs for the assay, were omitted).

\subsection{Data Analysis}

The data set of 214 SNPs was sorted according to predicted positions on the L. maculans supercontigs, as outlined in Supplementary Figure S1 of Rouxel et al. (2011). In order to look for potential SNP blocks relating to a parameter, the isolates were sorted individually, based on each parameter eg. State, stubble species or stubble cultivar (Table 1). Manhattan plots generated using the R package 'Gapit' (Lipka et al., 2012), were used to visualise any possible association between SNPs and these parameters.

All isolates were considered to be part of one population for the statistical analyses. The SNP positions were given 1 and 0 values (for the dendrogram and PCA analyses) or ' $\mathrm{A} / \mathrm{A}$ ' and ' $\mathrm{B} / \mathrm{B}$ ' (for $\mathrm{LD}$ analysis) for each genotype call 
and 'NA' was assigned to missing values. A binary distance matrix was generated and used to create a phylogenetic dendrogram. The R package "pvclust" (Suzuki \& Shimodaira, 2006) was used to generate a dendrogram with 1000 bootstrap iterations, binary distance and complete clustering. Population LD was calculated using the R package 'genetics' (Warnes, Gorjanc, Leisch, \& Man, 2012). $\mathrm{R}^{2} \mathrm{LD}$ values were used to generate the heatmap using the R package 'LDHeatmap' (Shin, Blay, McNeney, \& Graham, 2006) to visualise LD. PCA was performed using the R packages 'ade4' (Dray \& Dufour, 2007) and 'maptools' (Lewin-Koh et al., 2012). SNPs with possible null and private alleles were checked against the reference (Rouxel et al., 2011) using the alignment tool in Geneious Pro version 5.6 (Biomatters Ltd., 2015; Kearse et al., 2012).

\section{Results}

\subsection{Illumina GoldenGate results}

The results from the GoldenGate assay supported the SNP prediction of Zander et al. (2013). The data generated was sorted for SNPs that had high confidence clusters. 2.6\% of SNPs had missing values (NA) for all isolates, $29.9 \%$ were monomorphic and $11.7 \%$ were non-clustering SNPs. SNPs belonging to these three categories were eliminated from further analyses. No correlation between eliminated SNPs and SNP score or supercontig on which they were positioned could be observed. Filtering for quality polymorphic data resulted in a dataset of 214 SNPs. A subset of 193 SNPs was used for linkage disequilibrium (LD) after elimination of a further 21 SNPs. Reproducibility less than $100 \%$ was due only to missing data in one or other of the replicates.

Table 2. Percent reproducibility of replicates used in the assay

\begin{tabular}{ccc}
\hline Isolate name & Replicates & Reproducibility (\%) \\
\hline Lm-1 & 2 & 99.22 \\
Lm-2 & 2 & 98.96 \\
Lm-27 & 1 & 98.96 \\
Lm-32 & 1 & 99.74 \\
Lm-33 & 3 & 97.92 \\
Lm-34 & 3 & 92.52 \\
Lm-35 & 1 & 99.22 \\
Lm-36 & 3 & 97.20 \\
Lm-37 & 3 & 96.26 \\
Lm-38 & 3 & 96.26 \\
Lm-39 & 3 & 93.93 \\
Lm-40 & 3 & 92.06 \\
Lm-41 & 3 & 94.39 \\
Lm-46 & 1 & 95.57 \\
Lm-48 & 1 & 98.44 \\
Lm-49 & 1 & 98.96 \\
Lm-52 & 1 & 97.40 \\
Lm-54 & 1 & 98.96 \\
Lm-56 & 1 & 99.22 \\
\hline
\end{tabular}

\subsection{General Marker and Population Statistics}

The SNP data was mined for possible private alleles (Table 3). Private alleles are unique alleles in isolates that denote genetic distinctiveness. Of the 21 private allele SNPs, eight occurred in the isolate Lm- 1 and seven occurred in the isolate $\mathrm{Lm}-2$, both of which were used for SNP discovery. Of the other four alleles occurring at low frequency, four were in Lm-2 and Lm-55 only, and two were present in only Lm-2 and Lm-24 and Lm-2 and $\mathrm{Lm}-30$. SNPs private in $\mathrm{Lm}-2$ and $\mathrm{Lm}-55$ were the only ones that consistently occurred in intergenic regions of the genome. The SNP polymorphic information content (PIC) scores (See Appendix B) ranged from 0.3-0.5, indicative of relatively high polymorphism. General statistics can be found in Appendix B and association analysis of SNPs to the state the isolates were collected from can be found in Figures C1-C4 of Appendix C. 
Table 3. Possible private alleles identified and their location in the L. maculans genome

\begin{tabular}{lllll}
\hline SNP name & SuperContig & Location $(\mathrm{bp})$ & Polymorphic in & Location in genome \\
\hline SNP 55 & SuperContig_2 & 1120505 & Lm-1 & Intergenic \\
SNP 81 & SuperContig_13 & 1254623 & Lm-1 & Intergenic \\
SNP 85 & SuperContig_13 & 1424634 & Lm-1 & Intergenic \\
SNP 115 & SuperContig_8 & 774901 & Lm-2 and Lm-55 & Intergenic \\
SNP 125 & SuperContig_10 & 992433 & Lm-2 and Lm-55 & Intergenic \\
SNP 131 & SuperContig_6 & 638539 & Lm-2 and Lm-30 & Intergenic \\
SNP 135 & SuperContig_11 & 578821 & Lm-2 & Intergenic \\
SNP 137 & SuperContig_11 & 970997 & Lm-2 and Lm-55 & Intergenic \\
SNP 140 & SuperContig_11 & 1524610 & Lm-1 & End of SC \\
SNP 141 & SuperContig_3 & 95443 & Lm-2 & In gene similar to peroxisomal membrane \\
& & & & protein CBX93615.1 \\
SNP 151 & SuperContig_4 & 346457 & Lm-2 and Lm-55 & Intergenic \\
SNP 153 & SuperContig_4 & 873687 & Lm-1 & Downstream of gene product similar to \\
& & & & lipolytic \\
& & & & CBX9tein G-D-S-L family \\
SNP 160 & SuperContig_4 & 1358095 & Lm-2 & Intergenic \\
SNP 161 & SuperContig_4 & 1362010 & Lm-2 & Intergenic \\
SNP 181 & SuperContig_9 & 796976 & Lm-1 & Intergenic \\
SNP 188 & SuperContig_14 & 264857 & Lm-2 & Hypothetical protein CDS CBX98019.1 \\
SNP 194 & SuperContig_14 & 1268037 & Lm-1 & Hypothetical protein CDS CBX98389.1 \\
SNP 198 & SuperContig_16 & 447349 & Lm-1 & Exon of gene whose product is similar to \\
& & & & epoxide hydrolase CBX97267.1 \\
SNP 203 & SuperContig_17 & 350439 & Lm-2 & Hypothetical protein CDS CBX96839.1 \\
SNP 204 & SuperContig_17 & 523022 & Lm-2 and Lm-24 & Hypothetical protein CDS CBX96888.1 \\
SNP 205 & SuperContig_17 & 757759 & Lm-2 & Intergenic \\
\hline
\end{tabular}

\subsection{Population Analysis}

No significant correlation was observed in the data between the SNPs and the isolate collection site, the stubble cultivar, resistance complement of the cultivar or the year the isolates were collected (Table 1). A low level of association was observed between SNP73 on SC 12 and B. napus (as the stubble species) compared to $B$. juncea cultivars as the stubble species (Figure 1). No association to state, stubble cultivar, year collected or place collected could be found (Appendix C Figures C1-C4; other data not shown).

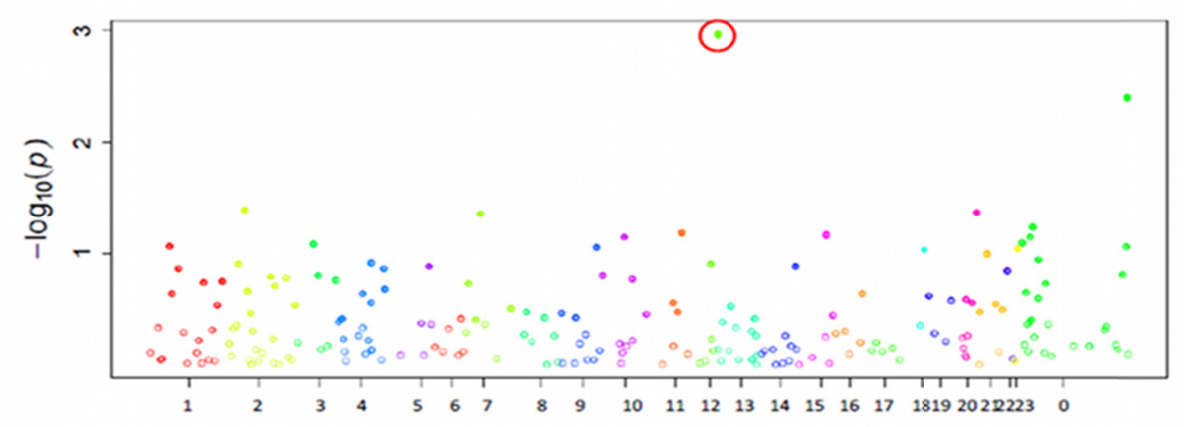

Figure 1. Manhattan plot of all isolates showing association between SNPs and B. napus species

Note: SNP 73 circled in red; $\mathrm{x}$-axis supercontigs; $\mathrm{y}$-xis $-\log 10$ values of association between stubble species and SNPs. 


\subsection{Phylogenetic Tree}

In order to visually analyse the relationships between the isolates, a dendrogram was generated. The isolates used for SNP prediction, Lm-1 and Lm-2 are on separate clades as can be discerned from the resulting phylogenetic tree (Figure 2). Based on this analysis, a number of isolates appeared to be genetically identical. DNA replicates used in the assay were noted to be the same (Table 2).

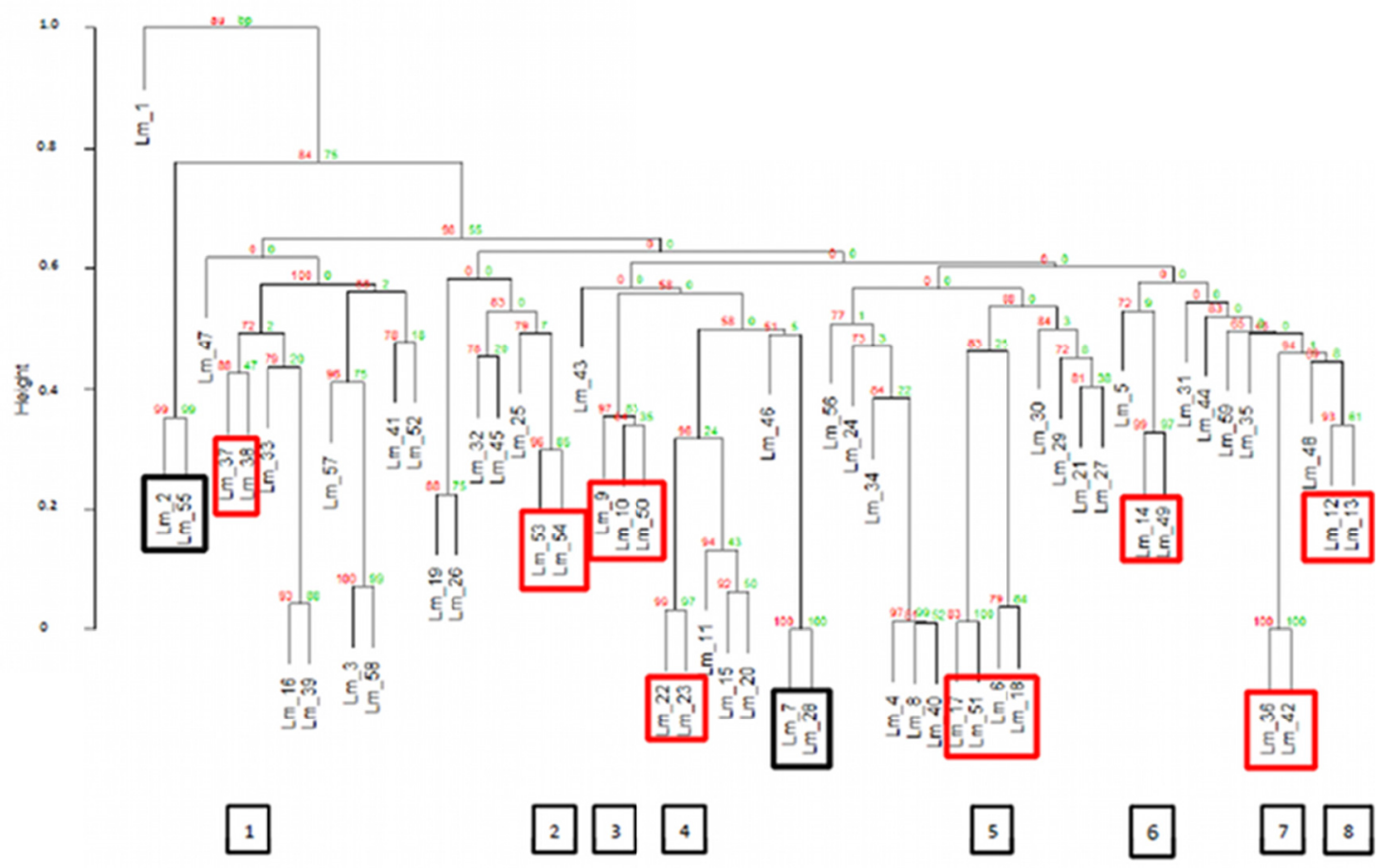

Figure 2. Phylogenetic tree based on all isolates

Note: Bootstrap values: red-Approximately Unbiased (AU) p-values calculated by multiscale bootstrap resampling; green-Bootstrap Probability (BP) p-values calculated by normal bootstrap resampling; AU values $>95 \%$ strongly supported by data (Suzuki \& Shimodaira, 2006); Replicates not shown; Left to right red boxes 1-8; Black boxes indicate similar isolates.

From the dendrogram it can be seen that the isolate Lm-55 was most similar to Lm-2 (Figure 2). Both isolates were collected in the same year (2006) and the same region of the state (Victoria) but from different stubble cultivars of B. napus. Isolates Lm-7 and Lm-28 were also seen to be similar. However, Lm-7 was collected in 2004 and Lm- 28 in 2003 from different stubble cultivars (Grace and ATR-Beacon respectively) and from different states (Wonwondah in Victoria and Moyhall in South Australia respectively, both close to a common border about 120 $\mathrm{km}$ apart). Boxes 1-8 denote isolates that group locally based on year collected, site of collection, state, stubble species and/or stubble cultivar (Figure 2), the details for which are seen in Table 1. The majority of clusters were from Victoria and South Australia. The most common groupings were based on state and stubble cultivar.

Some differences between certain isolates grouping together were also noticeable. Lm-16 and Lm-39 were isolated in different years $(2004,2005)$, from different stubble cultivars (ATR-Beacon, Surpass501TT) and in different places (Bordertown, SA and Mt. Barker, WA). The same differences could be seen for Lm-41 and Lm-52, Lm-32 and Lm-45, Lm15 and Lm-20, Lm-4, Lm-8 and Lm-40, Lm-6 and Lm-18 and Lm-21 and Lm-27. Therefore, small groupings based on the parameters listed in Table 1 were seen throughout the tree along with larger differences. No other patterns could be elicited from the positioning of sub-clades in this tree.

\subsection{Principal Component Analysis (PCA)}

The Principal Component Analysis (PCA) primarily showed a random distribution of isolates along the two principal component axes (Figure 3). Isolates Lm-1 and Lm-2 were completely different to each other, as expected 
based on their use for identification of polymorphic SNPs for designing the assay. Genetically identical isolates were plotted at the same point or grouped together in the same area, validating the results of the dendrogram (Figure 2). Isolates Lm-51 and Lm-17 were both collected in 2005 from South Australia. Isolates Lm-9 and Lm-10 were collected in 2004 isolated from "Grace" in Wonwondah. No other conclusive correlations could be elucidated.

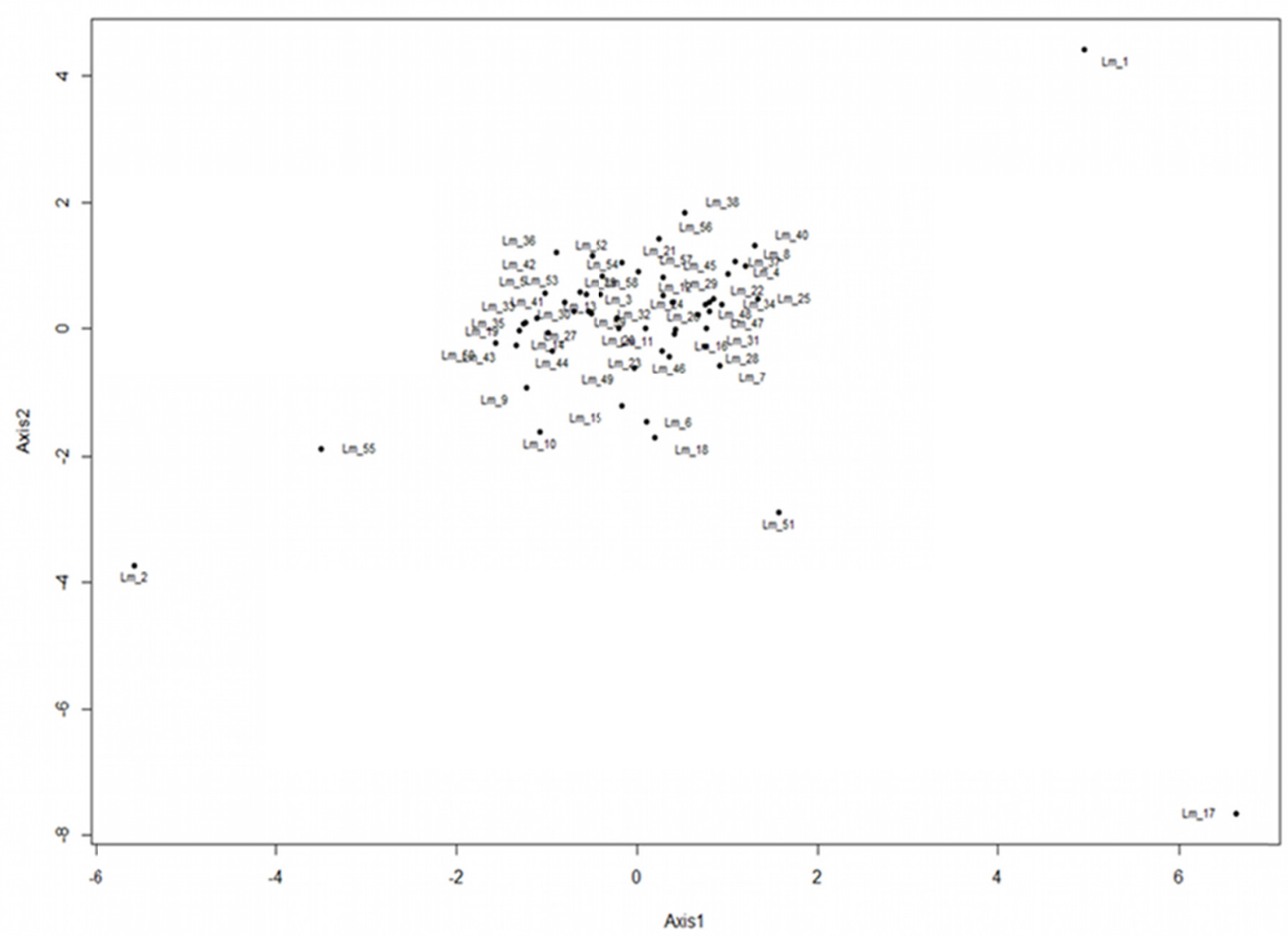

Figure 3. PCA displaying correlation between Australian L. maculans population isolates across 214 SNP loci isolates

Note: Axis1-Principal Component 1, Axis2-Principal Component 2; SNP prediction based on Lm-1 and Lm-2; replicates not shown.

\subsection{Linkage Disequilibrium}

The sub-data set of 193 SNPs, after elimination of 21 SNPs considered private alleles, was analysed to measure pairwise linkage disequilibrium (LD) between SNPs. The heatmap in Figure 4 displays results of the LD calculation. Based on the $\mathrm{R}^{2}$ values displayed in the heatmap, little significant $\mathrm{LD}$ was observed in the L. maculans population. $\mathrm{R}^{2}=1$ indicates no recombination, and thus high $\mathrm{LD}$, and $\mathrm{R}^{2}=0$ indicates considerable recombination thus no LD. Only $0.83 \%$ of p-values associated with pairwise comparisons were significant. Furthermore, the heatmap failed to show any noticeable patterns or blocks of $\mathrm{LD}$. 
Pairwise LD

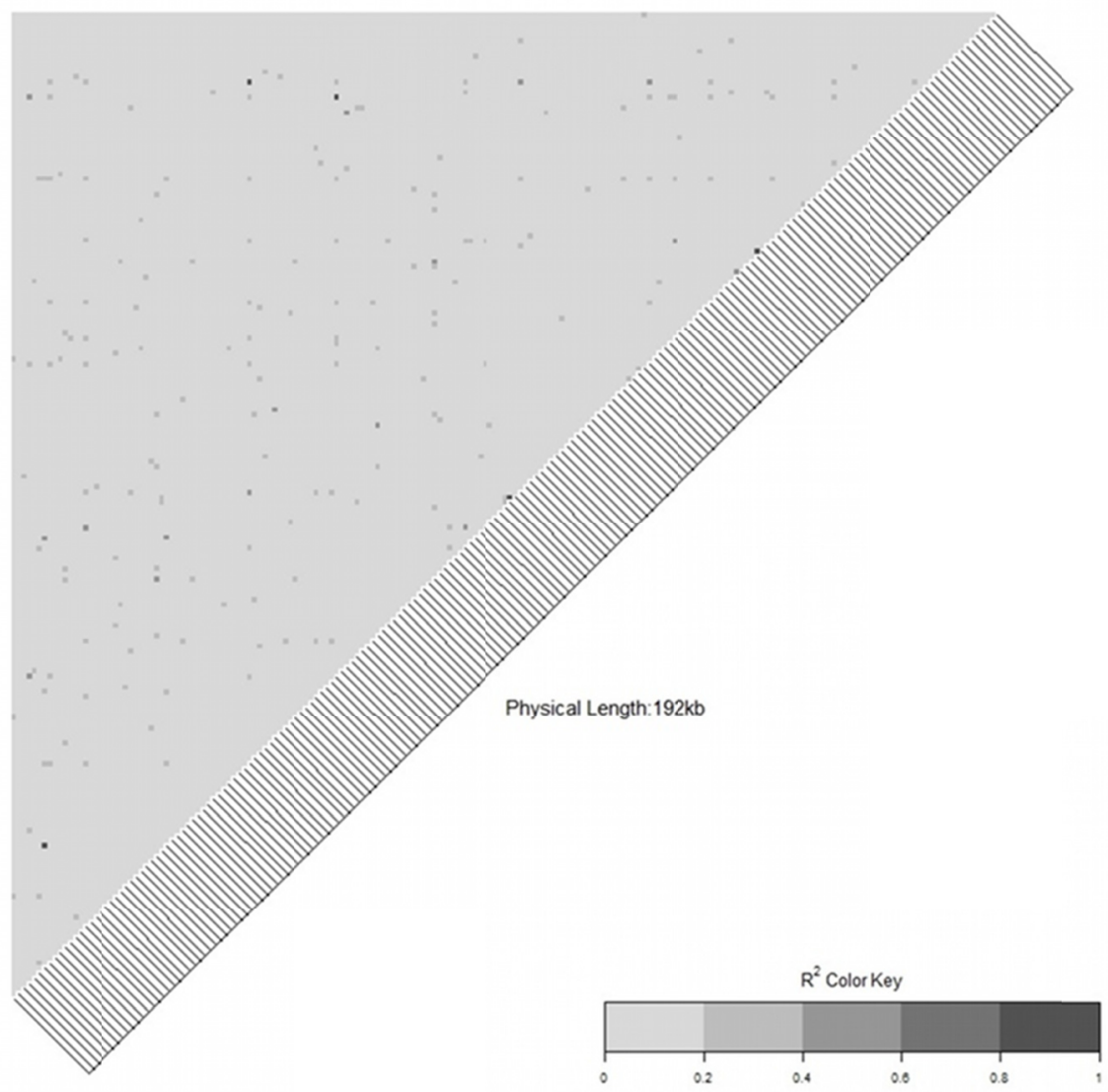

Figure 4. Heatmap displaying Linkage disequilibrium in Australian L. maculans population isolates across 193 SNP loci

Note: $\mathrm{R}^{2}=0$ considerable recombination; $\mathrm{R}^{2}=1$ no recombination.

\section{Discussion}

The isolates used in this study were collected from all around Australia. The absence of any obvious genetic variance specific to a certain parameter suggests that the Australian $L$. maculans isolates comprise a single population, but that some possible subpopulations localised to the state or site of collection do occur. Cultivar stubble may assist in maintaining the large population size (Daverdin et al., 2012; Travadon et al., 2011). No noticeable patterns or haplotypes were detected in the data, suggesting that this fungus evolves rapidly under selection pressure from the host. Sexual reproduction in this pathogen facilitates the production of ascospores which is its primary inoculum (Rouxel \& Balesdent, 2005). Human transport may aid in transporting infected material to different regions (Travadon et al., 2011). This in turn leads to random mating between isolates from different regions, creating genetic variance at the avirulence loci and assisting the pathogen to overcome host resistance (Dilmaghani et al., 2012).

It is expected that the population of a sexual reproductively active pathogen will be panmictic. In a panmictic population, members may interact with one another at random, which creates extensive recombination and genetic diversity (Polk \& Peek, 2010). Previous L. maculans population studies have reached the same conclusion of panmixia (Barrins et al., 2004; Travadon et al., 2011). We assumed the null hypothesis of a panmictic blackleg population across Australia while conducting population analyses, which was supported by the results of the manhattan plots, dendrogram, principal component analysis and linkage disequilibrium analysis.

Overall, the phylogenetic tree and PCA analysis suggested that L. maculans possesses a high evolutionary potential, indicative of populations able to overcome genetic resistance (McDonald \& Linde, 2002). Large population size, high rate of mutation, high genotype flow and mixed reproduction all confer high evolutionary potential to the pathogen, putatively enabling it to overcome host genetic resistance (McDonald \& Linde, 2002). The length of the tree branches indicates genetic similarity between isolates. Based on this, isolates Lm- 28 and 
Lm-7 appear to be genetically identical with $78 \%$ shared alleles (22\% missing values). These isolates have been collected from Moyhall, South Australia in 2003 and Wonwondah, Victoria in 2004 respectively. Van de Wouw et al. (2010) also used isolates Lm-6, Lm-17, Lm-18 and Lm-51 for genotyping at the AvrLm 1 and AvrLm6 loci. They classified Lm-18 and Lm-51 as haplotype 24, Lm-17 as haplotype 10 and Lm-6 as haplotype 4 based on their Avr genotype. These haplotypes displayed a completely different association in their study as compared to the dendrogram. This highlights the efficacy of using a large number of SNPs chosen from across the genome to classify the relationships between isolates.

Some local groupings based on state, year collected, stubble species, stubble cultivar and/or site of collection were also observed. Isolates in Box 1-8 (Figure 2) all clustered for certain parameters, with each cluster group sharing a single state of collection. Local groupings such as these may indicate the presence of small clonal subpopulations of this pathogen within Australia, such as were observed by Dilmaghani et al. (2013) on B. oleracea in Mexico. Clusters in our study were spread across the tree, indicating genetic differences between these possible subpopulations. Different conditions particular to each state such as weather, cultivars grown and stubble resistance all cumulatively affect the evolution of this pathogen. Therefore, it may be that conditions particular to each state promote asexual reproduction rather than sexual reproduction leading to less diversity within each subpopulation. Dilmaghani et al. (2013) attributed clonality such as this to moving the pathogen from its native biogeographic range, loss of a mating-type by mutation and culture conditions conducive to large-scale dispersal of conidia. This conclusion was made based on the presence of high linkage disequilibrium. Certain isolates like $\mathrm{Lm}-16$ and Lm-39 also clustered together but were vastly different in the parameters associated with them. It is known that human movement transports and introduces infected seed and plant material from one area to another (Dilmaghani et al., 2012) thereby mixing and changing the population, further attributing to its panmictic nature. The bootstrapping of the phylogenetic tree also supports the theory of a randomly interacting mixed population. Bootstrapping values for the main branches were $<95 \%$, which indicates low confidence in the hierarchical cluster analysis. On the other hand, most bootstrapping values within each box were $>95 \%$, indicating that they were strongly supported by the data (Suzuki \& Shimodaira, 2006). An overall analysis of the tree yielded no particular association to any other parameter. The PCA results also displayed a random positioning of isolates. Certain isolates clustered together, such as Lm-36 and Lm-42 isolates in Box 7, validating the dendrogram and also supporting panmixia. These findings could be attributed to the high evolutionary potential of L. maculans. Spore dispersal also plays a role in increasing gene flow and generating a random mix of isolates across the population (Travadon et al., 2011). More samples from each region and year will need to be collected and examined to investigate the possibility of clonal sub-populations of L. maculans within Australia.

We hypothesised that a possible cause of LD in this population could be selection of AvrLm genes, due to their impact on host plant infection. This would lead to loci in the selected region segregating with each other more often than expected by chance and can be visualised as blocks on the LD heatmap. However, we failed to notice any such patterns. As the rate of recombination between loci increases, there is a greater chance of linkage equilibrium in the population, decreasing LD. Populations that are constantly recombining and have a high cross-over rate will show little LD (McVean, 2008). Xu (2006) stated that only 5\% of locus-pairs have significant observed association to those expected in a completely panmictic population. Our LD analysis showed $0.83 \%$ of p-values associated with pairwise comparisons, to be significant. SNP73, which was seen to be significantly associated with B. napus cultivars and was located near Avr4-7, did not display significant LD. The SNP and the gene on $\mathrm{SC} 12$ of the L. maculans genome are $253.6 \mathrm{~kb}$ apart and the $\mathrm{GC}$ content of the traversing region is $45.2 \%$. Parlange et al. (2009) reported two PCR markers on the border of the AvrLm4-7 locus; the GC content between those markers was $35.2 \%$. It has been concluded by Rouxel et al. (2011) that recombination in the L. maculans genome occurs more frequently within GC-rich regions than between GC-rich regions. Therefore we believe that recombination events in the GC-rich region between the SNP and the gene may have impacted the association between them in B. napus cultivars. The number of samples isolated from B. napus cultivars may also be too small to clearly display this association on the heatmap in the form of LD. Furthermore, the majority of Australian cultivars that have been genotyped contain $R \operatorname{lm} 4$ and therefore there has been strong selection pressure at the AvrLm4-7 locus for a number of years in Australia (Marcroft et al., 2012). However, detailed studies comprising more isolates derived from $B$. juncea isolates will need to be conducted to confirm the validity of this association to B. napus cultivars. In the future, examining associations between SNPs and Avr genes in AT-rich regions such as these may prove fruitful in analysing the evolution of avirulence genes.

The results from this SNP genotyping assay successfully validated the work conducted by Zander et al. (2013) using the same SNP resource. The SNP prediction supported transferability of SNPs for use in the GoldenGate assay for the chosen SNPs. Stringent clustering criteria (ensuring that all SNPs visually separated into either the 'A' 
group or the 'B' group) yielded 214 SNPs, which were used for subsequent data analysis. Poor clustering could be a result of additional SNPs in the flanking regions of the predicted SNPs which can be resolved in the future by using more isolates for SNP discovery. Private alleles relating to five particular isolates (Lm-1, Lm-2, Lm-24, Lm-30 and Lm-55) were found. Lm-2 and Lm-55 were isolated from the same year and site of collection in Victoria. Alleles present only in the isolates used for SNP prediction (Lm-1 and Lm-2) are due to ascertainment bias of using these for the SNP prediction. Ascertainment bias is introduced because of the method used for SNP discovery (Albrechtsen, Nielsen, \& Nielsen, 2010), which in this case, used two isolates (Lm-1 and Lm-2) to predict SNPs. Ascertainment bias can be corrected in the future by using more isolates for SNP prediction. Being closely related and hence sharing sequence similarity might explain the four alleles that were found only in Lm-2 and Lm-55. The two main clades on the phylogenetic tree separated the isolates Lm-1 and Lm-2. Replicates used in this assay were seen to be genetically identical except for missing values. This was validated by the phylogenetic tree and PCA output, which confirmed the reproducibility of this assay.

The purpose behind conducting a large-scale genotyping assay was to understand the genetic diversity in the Australian L. maculans population. Our cumulative analysis of these results supports our null hypothesis of a panmictic Australian L. maculans population with possible regional clonality. This contrasts with the conclusions of Hayden et al. (2007) who found two genetically distinct eastern and western blackleg populations in Australia using 6 microsatellite and 2 minisatellite markers in 513 isolates collected over two years. The study also found $85 \%$ difference within the 13 subpopulations identified and $10 \%$ difference between the coasts. However, our results concur with the panmictic conclusion of Travadon et al. (2011). This study analysed 29 field populations of French L. maculans isolates using minisatellite markers and also found low genetic differentiation within populations. Further study concentrated on sampling from each region will help provide insights into this theory.

Overall, the high rate of sexual reproduction, ability of the pathogen to survive on stubble for long periods of time and random mating between isolates likely assist in maintaining a large blackleg population in Australia. This combined with its high evolutionary potential enables it to overcome host resistance quickly and cause infection leading to wide-spread crop losses. It is therefore imperative to attempt to restrict the population size of this pathogen using existing methods such as stubble management and introgression of resistance genes in Brassica species. Future work involves identifying new disease-associated genes, which will help in developing novel strategies to further control this devastating pathogen.

\section{Acknowledgments}

The authors would like to acknowledge funding support from the Australian Research Council (Projects LP0882095, LP0883462, LP0989200, LP110100200, DE120100668 and DP0985953). Support from the Australian Genome Research Facility (AGRF), the Queensland Cyber Infrastructure Foundation (QCIF) and the Australian Partnership for Advanced Computing (APAC) is gratefully acknowledged.

\section{References}

Albrechtsen, A., Nielsen, F. C., \& Nielsen, R. (2010). Ascertainment Biases in SNP chips affect measures of Population Divergence. Molecular biology and Evolution, 27(11), 2534-2547. http://dx.doi.org/10.1093/ molbev/msq148

Appleby, N., Edwards, D., \& Batley, J. (2009). New Technologies for Ultra-High Throughput Genotyping in Plants. Plant Genomics Methods and Protocols. UK: Humana Press. http://dx.doi.org/10.1007/978-1-59745 $-427-8 \_2$

Balesdent, M.H., Attard, A., Ansan-Melayah, D., Delourme, R., Renard, M., \& Rouxel, T. (2001) Genetic Control and Host Range of Avirulence toward Brassica napus Cultivars Quinta and Jet Neuf in Leptosphaeria maculans. Phytopathology, 91(1), 70-76. http://dx.doi.org/10.1094/PHYTO.2001.91.1.70

Balesdent, M. H., Barbetti, M. J., Li, H., Sivasithamparam, K., Gout, L., \& Rouxel, T. (2005). Analysis of Leptosphaeria maculans Race Structure in a Worldwide Collection of Isolates. Phytopathology, 95(9), 1061-1071. http://dx.doi.org/10.1094/PHYTO-95-1061

Barrins, J. M., Ades, P. K., Salisbury, P. A., \& Howlett, B. J. (2004). Genetic diversity of Australian Isolates of Leptosphaeria maculans, the Fungus that causes Blackleg of Canola (Brassica napus). Australasian Plant Pathology, 33(4), 529-536. http://dx.doi.org/10.1071/AP04061

Biomatters Ltd. (2015). Geneious Pro v5.6. Retrieved from http://www.geneious.com 
Daverdin, G., Rouxel, T., Gout, L., Aubertot, J. N., Fudal, I., Meyer, M., . . Balesdent, M. H. (2012). Genome Structure and Reproductive Behaviour influence the Evolutionary Potential of a Fungal Phytopathogen. Plos Pathogens, 8(11), e1003020. http://dx.doi.org/10.1371/journal.ppat.1003020

Dilmaghani, A., Gladieux, P., Gout, L., Giraud, T., Brunner, P. C., Stachowiak, A., . . . Rouxel, T. (2012). Migration Patterns and changes in Population Biology associated with the Worldwide Spread of the Oilseed Rape Pathogen Leptosphaeria maculans. Molecular Ecology, 21(10), 2519-2533. http://dx.doi.org/10.1111/j.1365-294X.2012.05535.x

Dilmaghani, A., Gout, L., Moreno-Rico, O., Dias, J. S., Coudard, L., Castillo-Torres, N., . . Rouxel, T. (2013). Clonal Populations of Leptosphaeria maculans Contaminating Cabbage in Mexico. Plant Pathology, 62(3), 520-532. http://dx.doi.org/10.1111/j.1365-3059.2012.02668.x

Dray, S., \& Dufour, A. B. (2007). The ade4 package: Implementing the Duality Diagram for Ecologists. Journal of Statistical Software, 22(4), 1-20.

Durstewitz, G., Polley, A., Plieske, J., Luerssen, H., Graner, E. M., Wieseke, R., \& Ganal, M. W. (2010). SNP discovery by amplicon sequencing and multiplex SNP genotyping in the allopolyploid species Brassica napus. Genome, 53(11). http://dx.doi.org/10.1139/G10-079

Fudal, I., Ross, S., Brun, H., Besnard, A.-L., Ermel, M., Kuhn, M.-L., . . Rouxel, T. (2009). Repeat-Induced Point Mutation (RIP) as an Alternative Mechanism of Evolution Toward Virulence in Leptosphaeria maculans. Molecular Plant-Microbe Interactions, 22(8), 932-941. http://dx.doi.org/10.1094/MPMI-22-8-0932

Giraud, T., Enjalbert, J., Fournier, E., Delmotte, F., \& Dutech, C. (2008). Population Genetics of Fungal Diseases of Plants. Parasite, 15(3), 449-454. http://dx.doi.org/10.1051/parasite/2008153449

Gout, L., Eckert, M., Rouxel, T., \& Balesdent, M. H. (2006). Genetic Variability and Distribution of Mating Type Alleles in Field Populations of Leptosphaeria maculans from France. Applied and Environmental Microbiology, 72(1), 185-191. http://dx.doi.org/10.1128/AEM.72.1.185-191.2006

Hayden, H. L., Cozijnsen, A. J., \& Howlett, B. J. (2007). Microsatellite and Minisatellite analysis of Leptosphaeria maculans in Australia reveals Regional Genetic Differentiation. Phytopathology, 97(7), 879-887. http://dx.doi.org/10.1094/PHYTO-97-7-0879

Hayward, A., McLanders, J., Campbell, E., Edwards, D., \& Batley, J. (2012). Genomic Advances will herald New Insights into the Brassica: Leptosphaeria maculans Pathosystem. Plant Biology, 14(1), 1-10. http://dx.doi.org/10.1111/j.1438-8677.2011.00481.x

Illumina Inc. (2013). GenomeStudio Software. Retrieved from http://www.illumina.com/applications/ microarrays/microarray-software/genomestudio.html

Kearse, M., Moir, R., Wilson, A., Stones-Havas, S., Cheung, M., Sturrock, S., Buxton, S., Cooper, A., Markowitz, S., Duran, C., Thierer, T., Ashton, B., Mentjies, P., \& Drummond, A. (2012). Geneious Basic: an Integrated and Extendable Desktop Software Platform for the Organization and Analysis of Sequence Data. Bioinformatics, 28(12), 1647-1649. http://dx.doi.org/10.1093/bioinformatics/bts199

Lewin-Koh, N. J., Bivand, R., Pebesma, E. J., Archer, E., Baddeley, A., Bibiko, H., . . Turner, R. (2012). maptools: Tools for Reading and Handling Spatial Objects (Version R package version 0.8-20). Retrieved from http://CRAN.R-project.org/package=maptools

Life Technologies (2013). Qubit巴 2.0 Fluorometer. Retrieved from http://www.invitrogen.com/site/us/en/ home/brands/Product-Brand/Qubit/qubit-fluorometer.html

Lipka, A. E., Tian, F., Wang, Q., Peiffer, J., Li, M., Bradbury, P. J., . . Z Zhang, Z. (2012). GAPIT: Genome Association and Prediction Integrated tool. Bioinformatics, 28(18), 2397-2399. http://dx.doi.org/10.1093/ bioinformatics/bts 444

Marcroft, S. J., Elliott, V. L., Cozijnsen, A. J., Salisbury, P. A., Howlett, B. J., \& Van de Wouw, A. P. (2012). Identifying Resistance Genes to Leptosphaeria maculans in Australian Brassica napus Cultivars based on Reactions to Isolates with known Avirulence Genotypes. Crop \& Pasture Science, 63(4), 338-350. http://dx.doi.org/10.1071/CP11341

McDonald, B. A., \& Linde, C. (2002). Pathogen Population Genetics, Evolutionary Potential, and Durable Resistance. Annual Review of Phytopathology, 40, 349-379. http://dx.doi.org/10.1146/annurev.phyto.40. 120501.101443 
McVean, G. (2007) Linkage Disequilibrium, Recombination and Selection, in Handbook of Statistical Genetics, Third Edition, John Wiley \& Sons, Ltd, Chichester, UK. http://dx.doi.org/10.1002/9780470061619.ch27

Parlange, F., Daverdin, G., Fudal, I., Kuhn, M. L., Balesdent, M. H., Blaise, F., . . . Rouxel, T. (2009). Leptosphaeria maculans Avirulence Gene AvrLm4-7 confers a Dual Recognition Specificity by the Rlm4 and Rlm7 Resistance Genes of Oilseed Rape, and circumvents Rlm4-mediated Recognition through a Single Amino Acid Change. Molecular Microbiology, 71(4), 851-863. http://dx.doi.org/10.1111/j.1365-2958.2008. 06547.x

Polk, D. B., \& Peek, R. M., Jr. (2010). Helicobacter pylori: Gastric Cancer and beyond. Nature Reviews Cancer, 10(6), 403-414. http://dx.doi.org/10.1038/nrc2857

Purwantara, A., Barrins, J. M., Cozijnsen, A. J., Ades, P. K., \& Howle, B. J. (2000). Genetic Diversity of Isolates of the Leptosphaeria maculans species complex from Australia, Europe and North America using Amplified Fragment Length Polymorphism analysis. Mycological Research, 104(7), 772-781. http://dx.doi.org/10. 1017/S095375629900235X

Purwantara, A., Salisbury, P. A., Burton, W. A., \& Howlett, B. J. (1998). Reaction of Brassica juncea (Indian mustard) lines to Australian isolates of Leptosphaeria maculans under Glasshouse and Field Conditions. European Journal of Plant Pathology, 104(9), 895-902. http://dx.doi.org/10.1023/A:1008609131695

Rouxel, T., \& Balesdent, M. H. (2005). The Stem Canker (blackleg) Fungus, Leptosphaeria maculans, enters the Genomic Era. Molecular Plant Pathology, 6(3), 225-241. http://dx.doi.org/10.1111/j.1364-3703.2005.002 82.x

Rouxel, T., Grandaubert, J., Hane, J. K., Hoede, C., van de Wouw, A. P., Couloux, A., . . Howlett, B. J. (2011). Effector Diversification within Compartments of the Leptosphaeria maculans Genome affected by Repeat-Induced Point Mutations. Nature Communications, 2. http://dx.doi.org/10.1038/ncomms1189

Shin, J. H., Blay, S., McNeney, B., \& Graham, J. (2006). LDheatmap: An R Function for Graphical Display of Pairwise Linkage Disequilibria between Single Nucleotide Polymorphisms. Journal of Statistical Software, 16, code snippet 03 .

Suzuki, R., \& Shimodaira, H. (2006). Pvclust: an R Package for Assessing the Uncertainty in Hierarchical Clustering. Bioinformatics, 22(12), 1540-1542. http://dx.doi.org/10.1093/bioinformatics/btl117

Tindall, E. A., Petersen, D. C., Nikolaysen, S., Miller, W., Schuster, S. C., \& Hayes, V. M. (2010). Interpretation of Custom Designed Illumina Genotype Cluster Plots for Targeted Association Studies and Next-Generation Sequence Validation. BMC research notes, 3, 39. http://dx.doi.org/10.1186/1756-0500-3-39

Travadon, R., Sache, I., Dutech, C., Stachowiak, A., Marquer, B., \& Bousset, L. (2011). Absence of Isolation by Distance Patterns at the Regional Scale in the Fungal Plant Pathogen Leptosphaeria maculans. Fungal Biology, 115(7), 649-659. http://dx.doi.org/10.1016/j.funbio.2011.03.009

Van de Wouw, A. P., Cozijnsen, A. J., Hane, J. K., Brunner, P. C., McDonald, B. A., Oliver, R. P., \& Howlett, B. J. (2010). Evolution of Linked Avirulence Effectors in Leptosphaeria maculans is affected by Genomic Environment and Exposure to Resistance Genes in Host Plants. Plos Pathogens, 6(11). http://dx.doi.org/10.1371/journal.ppat.1001180

Warnes, G., Gorjanc, G., Leisch, F., \& Man, M. (2012). genetics: Population Genetics (Version R package version 1.3.7.). Retrieved from http://CRAN.R-project.org/package=genetics

West, J. S., Kharbanda, P. D., Barbetti, M. J., \& Fitt, B. D. L. (2001). Epidemiology and Management of Leptosphaeria maculans (phoma stem canker) on Oilseed Rape in Australia, Canada and Europe. Plant Pathology, 50(1), 10-27. http://dx.doi.org/10.1046/j.1365-3059.2001.00546.x

Xu, J. R. (2006). Fundamentals of Fungal Molecular Population Genetic Analysis. Current Issues in Molecular Biology, 8, 75-89.

Zander, M., Patel, D. A., Van de Wouw, A., Lai, K., Lorenc, M. T., Campbell, E., . . Batley, J. (2013). Identifying Genetic Diversity of Avirulence Genes in Leptosphaeria maculans using Whole Genome Sequencing. Functional \& Integrative Genomics, 13(3), 295-308. http://dx.doi.org/10.1007/s10142-013-0324-5 


\section{Appendices}

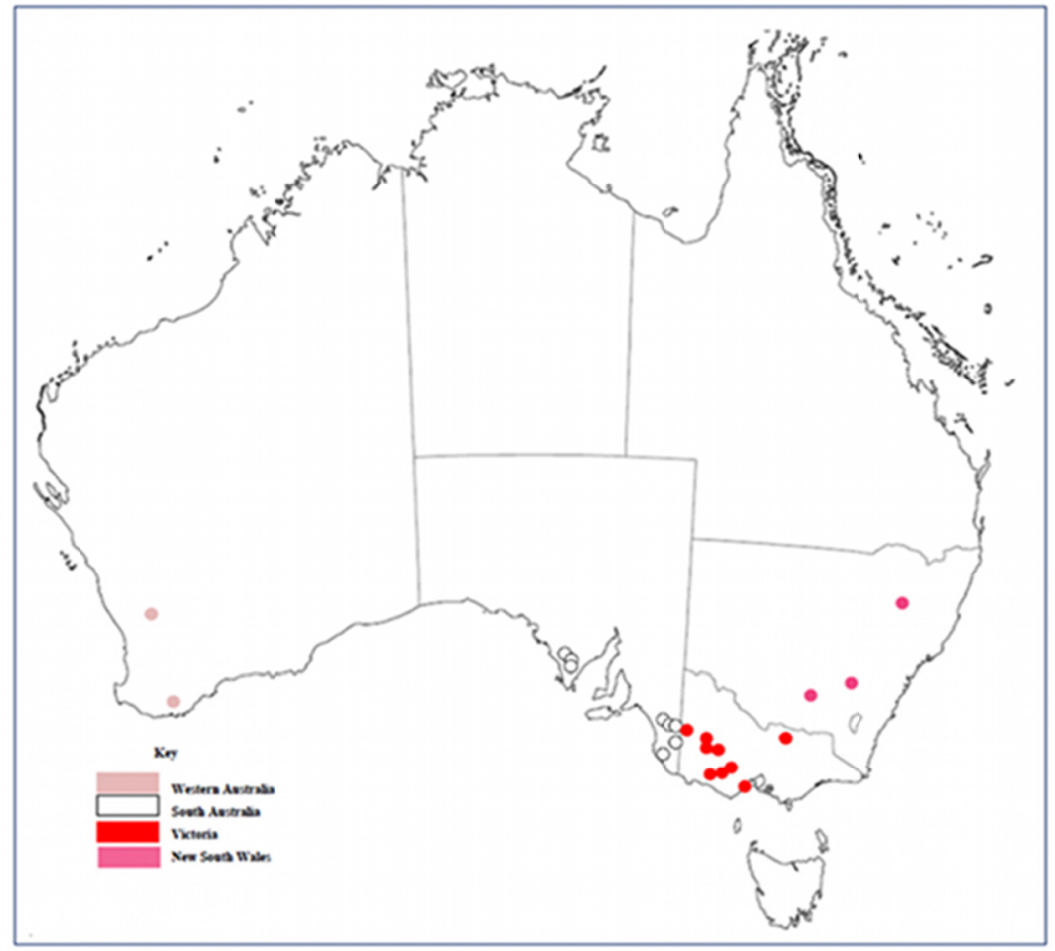

Appendix A. Site of collection of L. maculans in Australia

Appendix B. General statistics of 214 SNPs

\begin{tabular}{llllllll}
\hline SNP name & Total A & Total B & Total & NA & $\%$ A & $\%$ B & PIC \\
\hline SNP1 & 35 & 56 & 91 & 5 & 38.46 & 61.54 & 0.47 \\
SNP2 & 27 & 66 & 93 & 3 & 29.03 & 70.97 & 0.41 \\
SNP3 & 35 & 57 & 92 & 4 & 38.04 & 61.96 & 0.47 \\
SNP4 & 86 & 10 & 96 & 0 & 89.58 & 10.42 & 0.19 \\
SNP5 & 23 & 62 & 85 & 11 & 27.06 & 72.94 & 0.39 \\
SNP6 & 13 & 76 & 89 & 7 & 14.61 & 85.39 & 0.25 \\
SNP7 & 89 & 5 & 94 & 2 & 94.68 & 5.32 & 0.10 \\
SNP8 & 5 & 91 & 96 & 0 & 5.21 & 94.79 & 0.10 \\
SNP9 & 36 & 54 & 90 & 6 & 40.00 & 60.00 & 0.48 \\
SNP10 & 27 & 68 & 95 & 1 & 28.42 & 71.58 & 0.41 \\
SNP11 & 82 & 12 & 94 & 2 & 87.23 & 12.77 & 0.22 \\
SNP12 & 72 & 18 & 90 & 6 & 80.00 & 20.00 & 0.32 \\
SNP13 & 32 & 54 & 86 & 10 & 37.21 & 62.79 & 0.47 \\
SNP14 & 36 & 54 & 90 & 6 & 40.00 & 60.00 & 0.48 \\
SNP15 & 8 & 64 & 72 & 24 & 11.11 & 88.89 & 0.20 \\
SNP16 & 56 & 31 & 87 & 9 & 64.37 & 35.63 & 0.46 \\
SNP17 & 40 & 47 & 87 & 9 & 45.98 & 54.02 & 0.50 \\
SNP18 & 42 & 49 & 91 & 5 & 46.15 & 53.85 & 0.50 \\
SNP19 & 23 & 67 & 90 & 6 & 25.56 & 74.44 & 0.38 \\
SNP20 & 48 & 41 & 89 & 7 & 53.93 & 46.07 & 0.50 \\
SNP21 & 33 & 55 & 88 & 8 & 37.50 & 62.50 & 0.47 \\
SNP22 & 24 & 63 & 87 & 9 & 27.59 & 72.41 & 0.40 \\
SNP23 & 37 & 55 & 92 & 4 & 40.22 & 59.78 & 0.48 \\
SNP24 & 83 & 12 & 95 & 1 & 87.37 & 12.63 & 0.22 \\
SNP25 & 34 & 58 & 92 & 4 & 36.96 & 63.04 & 0.47 \\
\hline
\end{tabular}




\begin{tabular}{|c|c|c|c|c|c|c|c|}
\hline SNP26 & 19 & 68 & 87 & 9 & 21.84 & 78.16 & 0.34 \\
\hline SNP27 & 30 & 56 & 86 & 10 & 34.88 & 65.12 & 0.45 \\
\hline SNP28 & 16 & 75 & 91 & 5 & 17.58 & 82.42 & 0.29 \\
\hline SNP29 & 5 & 82 & 87 & 9 & 5.75 & 94.25 & 0.11 \\
\hline SNP30 & 35 & 58 & 93 & 3 & 37.63 & 62.37 & 0.47 \\
\hline SNP31 & 90 & 5 & 95 & 1 & 94.74 & 5.26 & 0.10 \\
\hline SNP32 & 52 & 40 & 92 & 4 & 56.52 & 43.48 & 0.49 \\
\hline SNP33 & 37 & 48 & 85 & 11 & 43.53 & 56.47 & 0.49 \\
\hline SNP34 & 73 & 23 & 96 & 0 & 76.04 & 23.96 & 0.36 \\
\hline SNP35 & 23 & 64 & 87 & 9 & 26.44 & 73.56 & 0.39 \\
\hline SNP36 & 80 & 12 & 92 & 4 & 86.96 & 13.04 & 0.23 \\
\hline SNP37 & 57 & 33 & 90 & 6 & 63.33 & 36.67 & 0.46 \\
\hline SNP38 & 35 & 50 & 85 & 11 & 41.18 & 58.82 & 0.48 \\
\hline SNP39 & 32 & 60 & 92 & 4 & 34.78 & 65.22 & 0.45 \\
\hline SNP40 & 28 & 67 & 95 & 1 & 29.47 & 70.53 & 0.42 \\
\hline SNP41 & 31 & 60 & 91 & 5 & 34.07 & 65.93 & 0.45 \\
\hline SNP42 & 29 & 56 & 85 & 11 & 34.12 & 65.88 & 0.45 \\
\hline SNP43 & 65 & 28 & 93 & 3 & 69.89 & 30.11 & 0.42 \\
\hline SNP44 & 35 & 58 & 93 & 3 & 37.63 & 62.37 & 0.47 \\
\hline SNP45 & 45 & 44 & 89 & 7 & 50.56 & 49.44 & 0.50 \\
\hline SNP46 & 38 & 52 & 90 & 6 & 42.22 & 57.78 & 0.49 \\
\hline SNP47 & 7 & 83 & 90 & 6 & 7.78 & 92.22 & 0.14 \\
\hline SNP48 & 9 & 84 & 93 & 3 & 9.68 & 90.32 & 0.17 \\
\hline SNP49 & 72 & 20 & 92 & 4 & 78.26 & 21.74 & 0.34 \\
\hline SNP50 & 74 & 20 & 94 & 2 & 78.72 & 21.28 & 0.33 \\
\hline SNP51 & 20 & 71 & 91 & 5 & 21.98 & 78.02 & 0.34 \\
\hline SNP52 & 44 & 47 & 91 & 5 & 48.35 & 51.65 & 0.50 \\
\hline SNP53 & 30 & 65 & 95 & 1 & 31.58 & 68.42 & 0.43 \\
\hline SNP54 & 77 & 16 & 93 & 3 & 82.80 & 17.20 & 0.28 \\
\hline SNP55 & 93 & 3 & 96 & 0 & 96.88 & 3.13 & 0.06 \\
\hline SNP56 & 20 & 71 & 91 & 5 & 21.98 & 78.02 & 0.34 \\
\hline SNP57 & 23 & 71 & 94 & 2 & 24.47 & 75.53 & 0.37 \\
\hline SNP58 & 30 & 59 & 89 & 7 & 33.71 & 66.29 & 0.45 \\
\hline SNP59 & 68 & 25 & 93 & 3 & 73.12 & 26.88 & 0.39 \\
\hline SNP60 & 74 & 20 & 94 & 2 & 78.72 & 21.28 & 0.33 \\
\hline SNP61 & 10 & 75 & 85 & 11 & 11.76 & 88.24 & 0.21 \\
\hline SNP62 & 74 & 16 & 90 & 6 & 82.22 & 17.78 & 0.29 \\
\hline SNP63 & 63 & 28 & 91 & 5 & 69.23 & 30.77 & 0.43 \\
\hline SNP64 & 6 & 89 & 95 & 1 & 6.32 & 93.68 & 0.12 \\
\hline SNP65 & 7 & 84 & 91 & 5 & 7.69 & 92.31 & 0.14 \\
\hline SNP66 & 46 & 43 & 89 & 7 & 51.69 & 48.31 & 0.50 \\
\hline SNP67 & 90 & 6 & 96 & 0 & 93.75 & 6.25 & 0.12 \\
\hline SNP68 & 76 & 17 & 93 & 3 & 81.72 & 18.28 & 0.30 \\
\hline SNP69 & 33 & 61 & 94 & 2 & 35.11 & 64.89 & 0.46 \\
\hline SNP70 & 75 & 20 & 95 & 1 & 78.95 & 21.05 & 0.33 \\
\hline SNP71 & 61 & 32 & 93 & 3 & 65.59 & 34.41 & 0.45 \\
\hline SNP72 & 88 & 7 & 95 & 1 & 92.63 & 7.37 & 0.14 \\
\hline SNP73 & 10 & 84 & 94 & 2 & 10.64 & 89.36 & 0.19 \\
\hline SNP74 & 29 & 60 & 89 & 7 & 32.58 & 67.42 & 0.44 \\
\hline SNP75 & 15 & 67 & 82 & 14 & 18.29 & 81.71 & 0.30 \\
\hline SNP76 & 49 & 43 & 92 & 4 & 53.26 & 46.74 & 0.50 \\
\hline SNP77 & 22 & 66 & 88 & 8 & 25.00 & 75.00 & 0.38 \\
\hline SNP78 & 46 & 43 & 89 & 7 & 51.69 & 48.31 & 0.50 \\
\hline SNP79 & 30 & 59 & 89 & 7 & 33.71 & 66.29 & 0.45 \\
\hline SNP80 & 17 & 78 & 95 & 1 & 17.89 & 82.11 & 0.29 \\
\hline SNP81 & 3 & 93 & 96 & 0 & 3.13 & 96.88 & 0.06 \\
\hline
\end{tabular}




\begin{tabular}{|c|c|c|c|c|c|c|c|}
\hline SNP82 & 55 & 31 & 86 & 10 & 63.95 & 36.05 & 0.46 \\
\hline SNP83 & 45 & 38 & 83 & 13 & 54.22 & 45.78 & 0.50 \\
\hline SNP84 & 57 & 39 & 96 & 0 & 59.38 & 40.63 & 0.48 \\
\hline SNP85 & 3 & 81 & 84 & 12 & 3.57 & 96.43 & 0.07 \\
\hline SNP86 & 6 & 43 & 49 & 47 & 12.24 & 87.76 & 0.21 \\
\hline SNP87 & 47 & 44 & 91 & 5 & 51.65 & 48.35 & 0.50 \\
\hline SNP88 & 81 & 14 & 95 & 1 & 85.26 & 14.74 & 0.25 \\
\hline SNP89 & 66 & 28 & 94 & 2 & 70.21 & 29.79 & 0.42 \\
\hline SNP90 & 35 & 58 & 93 & 3 & 37.63 & 62.37 & 0.47 \\
\hline SNP91 & 27 & 59 & 86 & 10 & 31.40 & 68.60 & 0.43 \\
\hline SNP92 & 14 & 64 & 78 & 18 & 17.95 & 82.05 & 0.29 \\
\hline SNP93 & 16 & 80 & 96 & 0 & 16.67 & 83.33 & 0.28 \\
\hline SNP94 & 84 & 8 & 92 & 4 & 91.30 & 8.70 & 0.16 \\
\hline SNP95 & 48 & 40 & 88 & 8 & 54.55 & 45.45 & 0.50 \\
\hline SNP96 & 84 & 11 & 95 & 1 & 88.42 & 11.58 & 0.20 \\
\hline SNP97 & 88 & 7 & 95 & 1 & 92.63 & 7.37 & 0.14 \\
\hline SNP98 & 36 & 58 & 94 & 2 & 38.30 & 61.70 & 0.47 \\
\hline SNP99 & 23 & 69 & 92 & 4 & 25.00 & 75.00 & 0.38 \\
\hline SNP100 & 18 & 67 & 85 & 11 & 21.18 & 78.82 & 0.33 \\
\hline SNP101 & 36 & 49 & 85 & 11 & 42.35 & 57.65 & 0.49 \\
\hline SNP102 & 36 & 49 & 85 & 11 & 42.35 & 57.65 & 0.49 \\
\hline SNP103 & 10 & 85 & 95 & 1 & 10.53 & 89.47 & 0.19 \\
\hline SNP104 & 42 & 50 & 92 & 4 & 45.65 & 54.35 & 0.50 \\
\hline SNP105 & 67 & 24 & 91 & 5 & 73.63 & 26.37 & 0.39 \\
\hline SNP106 & 39 & 55 & 94 & 2 & 41.49 & 58.51 & 0.49 \\
\hline SNP107 & 47 & 41 & 88 & 8 & 53.41 & 46.59 & 0.50 \\
\hline SNP108 & 5 & 91 & 96 & 0 & 5.21 & 94.79 & 0.10 \\
\hline SNP109 & 25 & 66 & 91 & 5 & 27.47 & 72.53 & 0.40 \\
\hline SNP110 & 24 & 65 & 89 & 7 & 26.97 & 73.03 & 0.39 \\
\hline SNP111 & 42 & 49 & 91 & 5 & 46.15 & 53.85 & 0.50 \\
\hline SNP112 & 45 & 48 & 93 & 3 & 48.39 & 51.61 & 0.50 \\
\hline SNP113 & 64 & 29 & 93 & 3 & 68.82 & 31.18 & 0.43 \\
\hline SNP114 & 22 & 69 & 91 & 5 & 24.18 & 75.82 & 0.37 \\
\hline SNP115 & 90 & 4 & 94 & 2 & 95.74 & 4.26 & 0.08 \\
\hline SNP116 & 46 & 42 & 88 & 8 & 52.27 & 47.73 & 0.50 \\
\hline SNP117 & 5 & 89 & 94 & 2 & 5.32 & 94.68 & 0.10 \\
\hline SNP118 & 54 & 39 & 93 & 3 & 58.06 & 41.94 & 0.49 \\
\hline SNP119 & 90 & 5 & 95 & 1 & 94.74 & 5.26 & 0.10 \\
\hline SNP120 & 47 & 32 & 79 & 17 & 59.49 & 40.51 & 0.48 \\
\hline SNP121 & 53 & 38 & 91 & 5 & 58.24 & 41.76 & 0.49 \\
\hline SNP122 & 28 & 63 & 91 & 5 & 30.77 & 69.23 & 0.43 \\
\hline SNP123 & 37 & 57 & 94 & 2 & 39.36 & 60.64 & 0.48 \\
\hline SNP124 & 11 & 84 & 95 & 1 & 11.58 & 88.42 & 0.20 \\
\hline SNP125 & 4 & 92 & 96 & 0 & 4.17 & 95.83 & 0.08 \\
\hline SNP126 & 16 & 74 & 90 & 6 & 17.78 & 82.22 & 0.29 \\
\hline SNP127 & 14 & 76 & 90 & 6 & 15.56 & 84.44 & 0.26 \\
\hline SNP128 & 7 & 53 & 60 & 36 & 11.67 & 88.33 & 0.21 \\
\hline SNP129 & 80 & 11 & 91 & 5 & 87.91 & 12.09 & 0.21 \\
\hline SNP130 & 92 & 4 & 96 & 0 & 95.83 & 4.17 & 0.08 \\
\hline SNP131 & 4 & 79 & 83 & 13 & 4.82 & 95.18 & 0.09 \\
\hline SNP132 & 49 & 39 & 88 & 8 & 55.68 & 44.32 & 0.49 \\
\hline SNP133 & 23 & 70 & 93 & 3 & 24.73 & 75.27 & 0.37 \\
\hline SNP134 & 66 & 24 & 90 & 6 & 73.33 & 26.67 & 0.39 \\
\hline SNP135 & 3 & 83 & 86 & 10 & 3.49 & 96.51 & 0.07 \\
\hline SNP136 & 76 & 16 & 92 & 4 & 82.61 & 17.39 & 0.29 \\
\hline SNP137 & 4 & 92 & 96 & 0 & 4.17 & 95.83 & 0.08 \\
\hline
\end{tabular}




\begin{tabular}{|c|c|c|c|c|c|c|c|}
\hline SNP138 & 91 & 5 & 96 & 0 & 94.79 & 5.21 & 0.10 \\
\hline SNP139 & 85 & 11 & 96 & 0 & 88.54 & 11.46 & 0.20 \\
\hline SNP140 & 92 & 3 & 95 & 1 & 96.84 & 3.16 & 0.06 \\
\hline SNP141 & 3 & 89 & 92 & 4 & 3.26 & 96.74 & 0.06 \\
\hline SNP142 & 24 & 70 & 94 & 2 & 25.53 & 74.47 & 0.38 \\
\hline SNP143 & 51 & 36 & 87 & 9 & 58.62 & 41.38 & 0.49 \\
\hline SNP144 & 40 & 47 & 87 & 9 & 45.98 & 54.02 & 0.50 \\
\hline SNP145 & 74 & 21 & 95 & 1 & 77.89 & 22.11 & 0.34 \\
\hline SNP146 & 73 & 15 & 88 & 8 & 82.95 & 17.05 & 0.28 \\
\hline SNP147 & 92 & 4 & 96 & 0 & 95.83 & 4.17 & 0.08 \\
\hline SNP148 & 9 & 86 & 95 & 1 & 9.47 & 90.53 & 0.17 \\
\hline SNP149 & 38 & 50 & 88 & 8 & 43.18 & 56.82 & 0.49 \\
\hline SNP150 & 65 & 29 & 94 & 2 & 69.15 & 30.85 & 0.43 \\
\hline SNP151 & 4 & 88 & 92 & 4 & 4.35 & 95.65 & 0.08 \\
\hline SNP152 & 31 & 61 & 92 & 4 & 33.70 & 66.30 & 0.45 \\
\hline SNP153 & 3 & 89 & 92 & 4 & 3.26 & 96.74 & 0.06 \\
\hline SNP154 & 29 & 58 & 87 & 9 & 33.33 & 66.67 & 0.44 \\
\hline SNP155 & 45 & 42 & 87 & 9 & 51.72 & 48.28 & 0.50 \\
\hline SNP156 & 19 & 71 & 90 & 6 & 21.11 & 78.89 & 0.33 \\
\hline SNP157 & 86 & 7 & 93 & 3 & 92.47 & 7.53 & 0.14 \\
\hline SNP158 & 66 & 24 & 90 & 6 & 73.33 & 26.67 & 0.39 \\
\hline SNP159 & 70 & 23 & 93 & 3 & 75.27 & 24.73 & 0.37 \\
\hline SNP160 & 93 & 3 & 96 & 0 & 96.88 & 3.13 & 0.06 \\
\hline SNP161 & 3 & 60 & 63 & 33 & 4.76 & 95.24 & 0.09 \\
\hline SNP162 & 74 & 20 & 94 & 2 & 78.72 & 21.28 & 0.33 \\
\hline SNP163 & 91 & 5 & 96 & 0 & 94.79 & 5.21 & 0.10 \\
\hline SNP164 & 75 & 17 & 92 & 4 & 81.52 & 18.48 & 0.30 \\
\hline SNP165 & 69 & 25 & 94 & 2 & 73.40 & 26.60 & 0.39 \\
\hline SNP166 & 56 & 35 & 91 & 5 & 61.54 & 38.46 & 0.47 \\
\hline SNP167 & 61 & 27 & 88 & 8 & 69.32 & 30.68 & 0.43 \\
\hline SNP168 & 69 & 24 & 93 & 3 & 74.19 & 25.81 & 0.38 \\
\hline SNP169 & 29 & 63 & 92 & 4 & 31.52 & 68.48 & 0.43 \\
\hline SNP170 & 30 & 55 & 85 & 11 & 35.29 & 64.71 & 0.46 \\
\hline SNP171 & 39 & 50 & 89 & 7 & 43.82 & 56.18 & 0.49 \\
\hline SNP172 & 75 & 17 & 92 & 4 & 81.52 & 18.48 & 0.30 \\
\hline SNP173 & 17 & 76 & 93 & 3 & 18.28 & 81.72 & 0.30 \\
\hline SNP174 & 41 & 47 & 88 & 8 & 46.59 & 53.41 & 0.50 \\
\hline SNP175 & 4 & 88 & 92 & 4 & 4.35 & 95.65 & 0.08 \\
\hline SNP176 & 26 & 65 & 91 & 5 & 28.57 & 71.43 & 0.41 \\
\hline SNP177 & 17 & 76 & 93 & 3 & 18.28 & 81.72 & 0.30 \\
\hline SNP178 & 9 & 79 & 88 & 8 & 10.23 & 89.77 & 0.18 \\
\hline SNP179 & 59 & 34 & 93 & 3 & 63.44 & 36.56 & 0.46 \\
\hline SNP180 & 33 & 59 & 92 & 4 & 35.87 & 64.13 & 0.46 \\
\hline SNP181 & 3 & 69 & 72 & 24 & 4.17 & 95.83 & 0.08 \\
\hline SNP182 & 25 & 69 & 94 & 2 & 26.60 & 73.40 & 0.39 \\
\hline SNP183 & 71 & 17 & 88 & 8 & 80.68 & 19.32 & 0.31 \\
\hline SNP184 & 22 & 69 & 91 & 5 & 24.18 & 75.82 & 0.37 \\
\hline SNP185 & 48 & 42 & 90 & 6 & 53.33 & 46.67 & 0.50 \\
\hline SNP186 & 81 & 14 & 95 & 1 & 85.26 & 14.74 & 0.25 \\
\hline SNP187 & 22 & 63 & 85 & 11 & 25.88 & 74.12 & 0.38 \\
\hline SNP188 & 3 & 92 & 95 & 1 & 3.16 & 96.84 & 0.06 \\
\hline SNP189 & 54 & 35 & 89 & 7 & 60.67 & 39.33 & 0.48 \\
\hline SNP190 & 13 & 79 & 92 & 4 & 14.13 & 85.87 & 0.24 \\
\hline SNP191 & 15 & 76 & 91 & 5 & 16.48 & 83.52 & 0.28 \\
\hline SNP192 & 70 & 21 & 91 & 5 & 76.92 & 23.08 & 0.36 \\
\hline SNP193 & 7 & 85 & 92 & 4 & 7.61 & 92.39 & 0.14 \\
\hline
\end{tabular}




\begin{tabular}{llllllll}
\hline SNP194 & 3 & 93 & 96 & 0 & 3.13 & 96.88 & 0.06 \\
SNP195 & 39 & 49 & 88 & 8 & 44.32 & 55.68 & 0.49 \\
SNP196 & 66 & 25 & 91 & 5 & 72.53 & 27.47 & 0.40 \\
SNP197 & 7 & 80 & 87 & 9 & 8.05 & 91.95 & 0.15 \\
SNP198 & 93 & 3 & 96 & 0 & 96.88 & 3.13 & 0.06 \\
SNP199 & 41 & 50 & 91 & 5 & 45.05 & 54.95 & 0.50 \\
SNP200 & 13 & 83 & 96 & 0 & 13.54 & 86.46 & 0.23 \\
SNP201 & 53 & 39 & 92 & 4 & 57.61 & 42.39 & 0.49 \\
SNP202 & 40 & 47 & 87 & 9 & 45.98 & 54.02 & 0.50 \\
SNP203 & 3 & 93 & 96 & 0 & 3.13 & 96.88 & 0.06 \\
SNP204 & 4 & 82 & 86 & 10 & 4.65 & 95.35 & 0.09 \\
SNP205 & 92 & 3 & 95 & 1 & 96.84 & 3.16 & 0.06 \\
SNP206 & 73 & 20 & 93 & 3 & 78.49 & 21.51 & 0.34 \\
SNP207 & 75 & 18 & 93 & 3 & 80.65 & 19.35 & 0.31 \\
SNP208 & 32 & 57 & 89 & 7 & 35.96 & 64.04 & 0.46 \\
SNP209 & 60 & 33 & 93 & 3 & 64.52 & 35.48 & 0.46 \\
SNP210 & 76 & 19 & 95 & 1 & 80.00 & 20.00 & 0.32 \\
SNP211 & 45 & 46 & 91 & 5 & 49.45 & 50.55 & 0.50 \\
SNP212 & 33 & 57 & 90 & 6 & 36.67 & 63.33 & 0.46 \\
SNP213 & 19 & 72 & 91 & 5 & 20.88 & 79.12 & 0.33 \\
SNP214 & 25 & 60 & 85 & 11 & 29.41 & 70.59 & 0.42 \\
\hline
\end{tabular}

Note: PIC-Polymorphism Information Content (Totals excluding NAs).

Appendix C. Analysis of association between SNPs and state of collection of isolates

\section{SA}

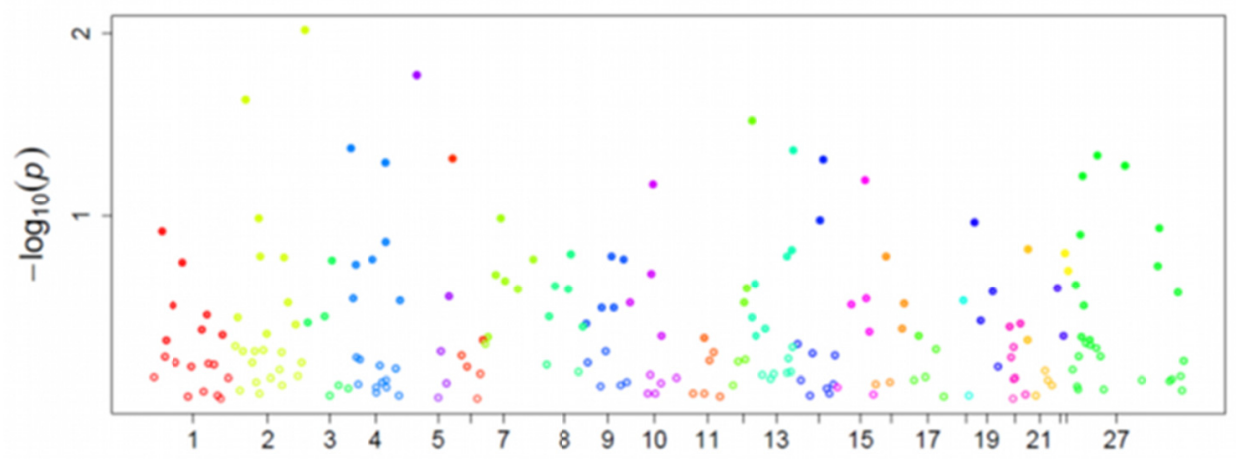

Figure C1: Manhattan plot of all isolates showing association between SNPs and South Australia Note: $\mathrm{x}$-axis: Supercontigs $(27=\mathrm{SC})$; $\mathrm{y}$-xis $-\log _{10}$ values of association between stubble species and SNPs.

\section{.NSW}

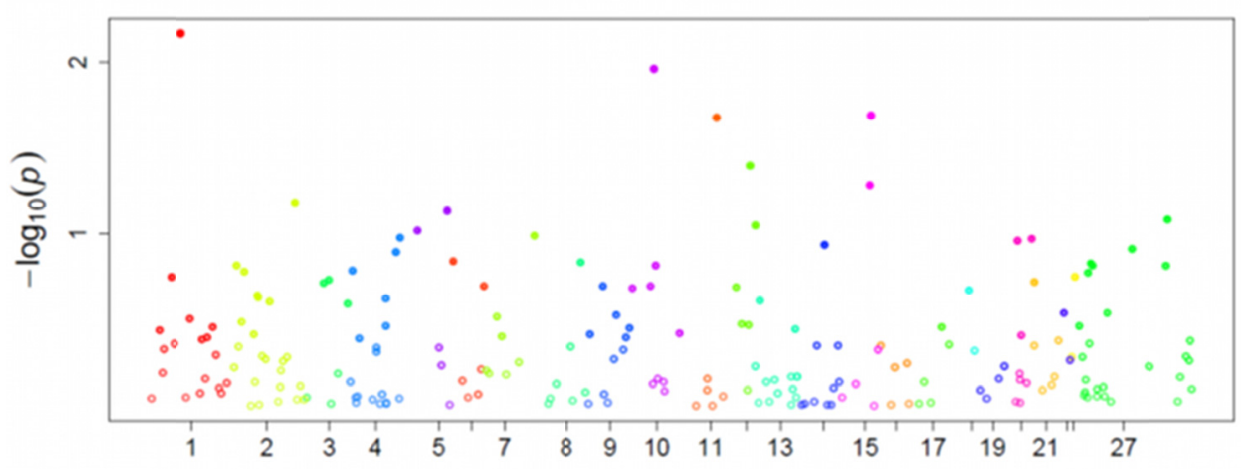

Figure C2: Manhattan plot of all isolates showing association between SNPs and New South Wales Note: $\mathrm{x}$-axis supercontigs $(27=\mathrm{SC} 0)$; $\mathrm{y}$-xis $-\log _{10}$ values of association between stubble species and SNPs. 


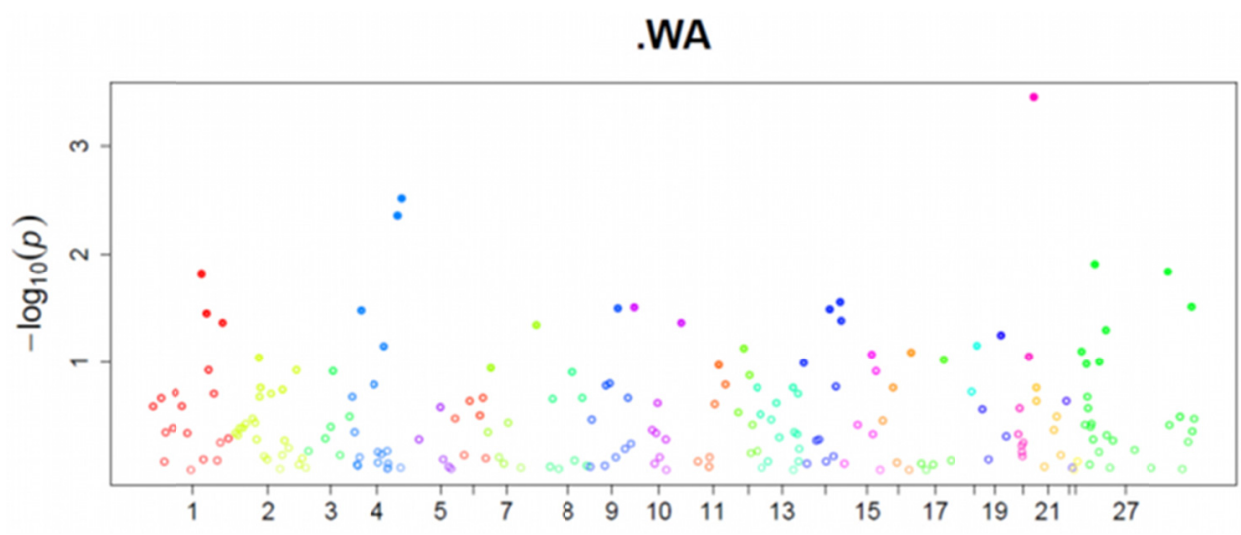

Figure C3 Manhattan plot of all isolates showing association between SNPs and Western Australia Note: $\mathrm{x}$-axis supercontigs $(27=\mathrm{SC})$; $\mathrm{y}$-xis $-\log _{10}$ values of association between stubble species and SNPs.

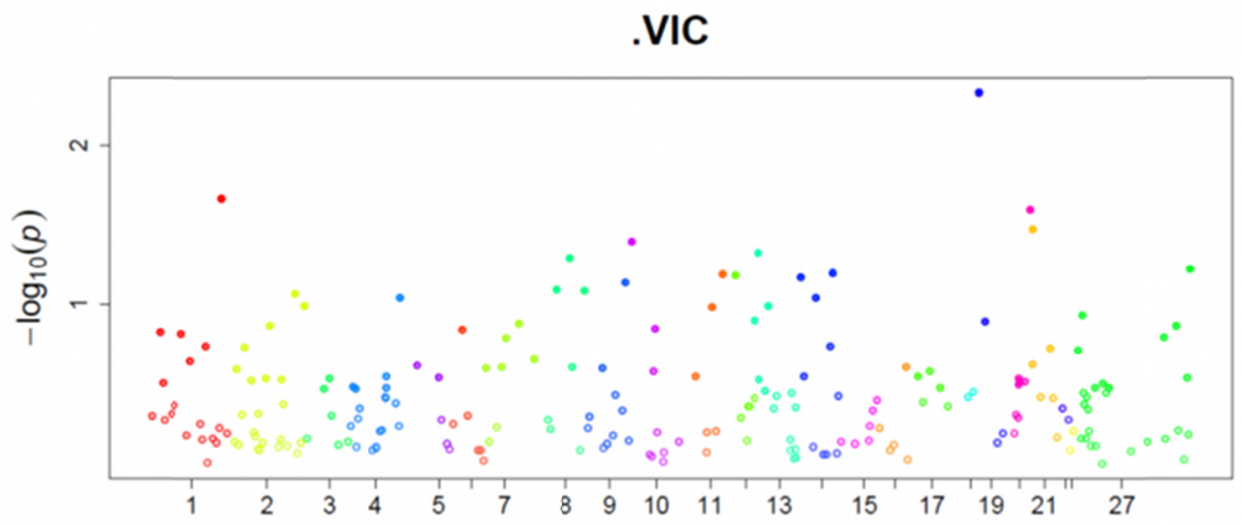

Figure C4 Manhattan plot of all isolates showing association between SNPs and Victoria

Note: $\mathrm{x}$-axis supercontigs $(27=\mathrm{SC})$; $\mathrm{y}$-xis $-\log _{10}$ values of association between stubble species and SNPs.

\section{Copyrights}

Copyright for this article is retained by the author(s), with first publication rights granted to the journal.

This is an open-access article distributed under the terms and conditions of the Creative Commons Attribution license (http://creativecommons.org/licenses/by/3.0/). 